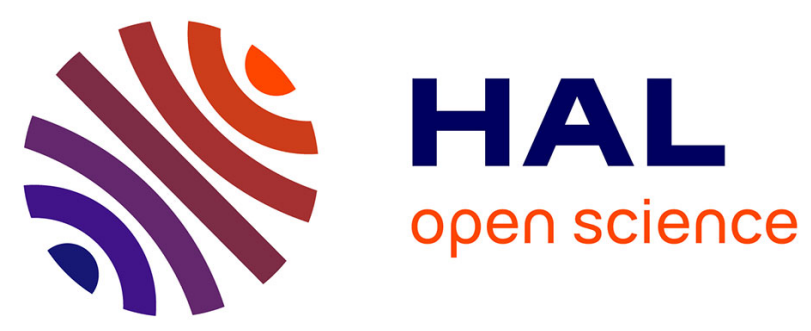

\title{
Online orientation distribution function reconstruction in constant solid angle and its application to motion detection in HARDI
}

Emmanuel Caruyer, Iman Aganj, Ryan Muetzel, Christophe Lenglet, Guillermo Sapiro, Rachid Deriche

\section{To cite this version:}

Emmanuel Caruyer, Iman Aganj, Ryan Muetzel, Christophe Lenglet, Guillermo Sapiro, et al.. Online orientation distribution function reconstruction in constant solid angle and its application to motion detection in HARDI. International Symposium on Biomedical Imaging: From Nano to Macro, Apr 2010, Rotterdam, Netherlands. inria-00559884

\section{HAL Id: inria-00559884 \\ https://inria.hal.science/inria-00559884}

Submitted on 26 Jan 2011

HAL is a multi-disciplinary open access archive for the deposit and dissemination of scientific research documents, whether they are published or not. The documents may come from teaching and research institutions in France or abroad, or from public or private research centers.
L'archive ouverte pluridisciplinaire HAL, est destinée au dépôt et à la diffusion de documents scientifiques de niveau recherche, publiés ou non, émanant des établissements d'enseignement et de recherche français ou étrangers, des laboratoires publics ou privés. 


\title{
ONLINE ORIENTATION DISTRIBUTION FUNCTION RECONSTRUCTION IN CONSTANT SOLID ANGLE AND ITS APPLICATION TO MOTION DETECTION IN HARDI
}

\author{
Emmanuel Caruyer ${ }^{1}$, Iman Aganj ${ }^{2}$, Ryan L. Muetzel ${ }^{3}$, \\ Christophe Lenglet $^{2,4}$, Guillermo Sapiro ${ }^{2}$, Rachid Deriche ${ }^{1}$ \\ ${ }^{1}$ Athena Project-Team, INRIA Sophia Antipolis - Méditerranée, France \\ ${ }^{2}$ Department of Electrical and Computer Engineering, University of Minnesota, Minneapolis, MN, USA \\ ${ }^{3}$ Department of Psychology, University of Minnesota, Minneapolis, MN, USA \\ ${ }^{4}$ Center for Magnetic Resonance Research, University of Minnesota, Minneapolis, MN, USA
}

\begin{abstract}
The diffusion orientation distribution function (ODF) can be reconstructed from $q$-ball imaging (QBI) to map the complex intravoxel structure of water diffusion. As acquisition time is particularly large for high angular resolution diffusion imaging (HARDI), fast estimation algorithms have recently been proposed, as an on-line feedback on the reconstruction accuracy. Thus the acquisition could be stopped or continued on demand. We adapt these real-time algorithms to the mathematically correct definition of ODF in constant solid angle (CSA), and develop a motion detection algorithm upon this reconstruction. Results of improved fiber crossing detection by CSA ODF are shown, and motion detection was implemented and tested in vivo.
\end{abstract}

Index Terms - On-line model estimation, high angular resolution diffusion imaging (hardi), q-ball imaging (qbi), orientation distribution function (odf), motion estimation.

\section{INTRODUCTION}

Diffusion Magnetic Resonance Imaging (MRI) is a recent technological advance which has rapidly been considered by the medical community as a help for diagnosis and preoperative planning. By measuring the local diffusion properties of water molecules, it allows to infer underlying tissue structure and physiological properties. White matter fiber bundles are especially well suited to this analysis. By mapping the Orientation Distribution Function (ODF), i.e. the angular information of the diffusion Probability Density Function (PDF), $q$-Ball Imaging (QBI) [1] can resolve complex intravoxel structure. So as to speed up the reconstruction, analytical computation of the ODF has further been proposed [2], allowing ODF reconstruction in a clinical context. Recently, the commonly used ODF definition was corrected to match the definition of the marginal PDF of diffusion in a Constant Solid Angle (CSA) [3, 4]. This moves the community towards more accurate models, and more flexible algorithms to reconstruct these models.
An important limitation of the widespread adoption of diffusion MRI by clinicians remains its sensitivity to patient motion. It is indeed delicate to compensate patient motion through registration of the Diffusion Weighted Images (DWI) prior to model estimation [5]. A real-time (on-line) reconstruction algorithm has recently been proposed [6, 7], together with an acquisition sequence designed to be optimal whenever the scan is interrupted. This is based on a Kalman filter adaptive approach. In this work, we extend the framework proposed in [7]: we adapt it to reconstruct the CSA ODF, and show how to use this as a motion detection tool during acquisition.

In Section 2, we review the estimation of CSA ODF and present the subsequent changes in the Kalman filter implementation. We also propose a solution to the motion detection based on the incremental estimation of the diffusion signal. Section 3 presents experimental results of fiber crossing detection from synthetic and real data, comparing the original ODF to the CSA ODF, and results on how motion is readily detected in vivo with our method. Section 4 concludes with a review of the contributions.

\section{MATERIAL AND METHODS}

\subsection{Kalman filtering with ODF in constant solid angle}

The ODF has originally been defined and estimated in QBI as [1]:

$$
\operatorname{ODF}_{\text {orig. }}(\hat{u}):=\frac{1}{Z} \int_{0}^{\infty} P(r \hat{u}) \mathrm{d} r \approx \frac{1}{Z} \operatorname{FRT}\{S(\hat{u})\}
$$

with $P(r \hat{u})$ the $3 \mathrm{D}$ probability function (PDF) of the diffusion of water molecules, $S(\hat{u})$ the diffusion signal in the direction $\hat{u}$, FRT the Funk-Radon transform $[1,8]$, and $Z$ the normalization constant which needs to be computed. $Z$ is required because, by lacking the Jacobian factor $r^{2}$, the above definition of ODF is not the actual marginal PDF of diffusion in a given direction in CSA (see [3, 4]). It was shown in [3] that, 
by considering the Jacobian factor, the following expression for the CSA ODF can be derived, better resolving multiple fiber orientations:

$$
\begin{aligned}
\operatorname{ODF}_{\mathrm{CSA}}(\hat{u}) & :=\int_{0}^{\infty} P(r \hat{u}) r^{2} \mathrm{~d} r \\
& \approx \frac{1}{4 \pi}+\frac{1}{16 \pi^{2}} \mathrm{FRT}\left\{\nabla_{b}^{2} \ln (-\ln E(\hat{u}))\right\}
\end{aligned}
$$

where $E(\hat{u}):=S(\hat{u}) / S_{0}$ with $S_{0}$ the baseline image, and $\nabla_{b}^{2}$ the Laplace-Beltrami operator. The above dimensionless and intrinsically normalized formula obviates the need for postprocessing such as manual normalization or artificial sharpening. The framework has been extended to multiple $q$-shells in [3].

A robust implementation of the original ODF was proposed in $[2,9,10]$, which uses a least square error scheme to approximate the signal in the spherical harmonic $(\mathrm{SH})$ basis, and then computes the FRT analytically. This method has also been exploited in [3] to calculate the CSA ODF, where $\ln (-\ln E(\hat{u}))$, instead of $S(\hat{u})$, is approximated in the $\mathrm{SH}$ basis and the FRT and $\nabla_{b}^{2}$ operators are afterwards computed analytically. Additionally, [2] introduces a regularization term proven to be useful especially when dealing with noisy data.

While this implementation scheme can be very efficient for offline computation of the ODFs, the required pseudoinversion may be computationally too expensive for real-time reconstruction of almost a million ODFs in a single HARDI scan. Besides, in online ODF estimation, we do not expect intuitively that the new measurement at each iteration bring such fundamental information as to necessitate re-solving the entire system of equations. Kalman filtering [11], is generally the first and simplest answer in such cases where we desire to minimize the computational cost of the real-time solution to a growing linear problem by making optimum use of the solution at each step to update it on the fly. A particular Kalman filtering framework has been proposed in [6] to incrementally compute the original QBI ODFs taking into account the regularization factor in [2]. Recently, the authors of [7] showed the sub-optimality of [6] and suggested a new implementation of the method which does not compromise the optimality of the Kalman filter.

In this work we use the same approach as in [7], yet this time to compute the CSA ODFs [3] on-line. This is again done by SH approximation of the double logarithm of the signal, instead of the signal itself:

$$
\ln (-\ln E(\hat{u})) \approx \sum_{j=1}^{R} \hat{c}_{j} Y_{j}(\hat{u})
$$

where $\left\{Y_{j}, j=1, \ldots, R\right\}$ is the $l$-th order $\mathrm{SH}$ basis for real and symmetric functions on the unit sphere [2], $R=$ $(l+1)(l+2) / 2$ is the dimension of this basis, and $\hat{\mathbf{c}}=$ $\left(\hat{c}_{j}\right)_{j=1, \ldots, R}$ the corresponding vector of coefficients. An incremental computation of the coefficients $\hat{\mathbf{c}}$ lets us reconstruct the CSA ODF at each step, in a similar way as done offline in [3]. The corresponding Kalman filter equations are:

$$
\left\{\begin{array}{l}
\hat{\mathbf{c}}_{0}=\mathbb{E}\left[\mathbf{c}_{0}\right] \\
\tilde{\mathbf{P}}_{0}=\mathbb{E}\left[\left(\mathbf{c}-\hat{\mathbf{c}}_{0}\right)\left(\mathbf{c}-\hat{\mathbf{c}}_{0}\right)^{T}\right] \\
\mathbf{P}_{0}=\left(\tilde{\mathbf{P}}_{0}^{-1}+\lambda \mathbf{L}\right)^{-1} \\
\mathbf{g}_{k}=\mathbf{P}_{k-1} \mathbf{X}_{k}^{T}\left(\mathbf{X}_{k} \mathbf{P}_{k-1} \mathbf{X}_{k}^{T}+\sigma_{k}^{2}\right)^{-1} \\
\mathbf{P}_{k}=\left(\mathbf{I}-\mathbf{g}_{k} \mathbf{X}_{k}\right) \mathbf{P}_{k-1} \\
\hat{\mathbf{c}}_{k}=\hat{\mathbf{c}}_{k-1}+\mathbf{g}_{k}\left(y_{k}-\mathbf{X}_{k} \hat{\mathbf{c}}_{k-1}\right)
\end{array}\right.
$$

where $y_{k}=\ln \left(-\ln E\left(\hat{u}_{k}\right)\right)$ is the double logarithm of the signal measured in the direction $\hat{u}_{k}, \mathbf{X}_{k}$ is the $k$-th row of the SH matrix $\mathbf{X}$, where $X_{k, j}=Y_{j}\left(\hat{u}_{k}\right) . \quad \mathbf{P}_{k}$ and $\mathbf{g}_{k}$ are standard notations for the Kalman covariance matrix and gain, and $\sigma_{k}^{2}$ is the covariance of the signal measured at iteration $k$. For more details on the parameters, vectors and matrices, we kindly refer the reader to [7].

Finally, the SH coefficients of the CSA ODF are computed as:

$$
\hat{c}_{j}^{\prime}=\left\{\begin{array}{cc}
\frac{1}{2 \sqrt{\pi}} & j=1 \\
-\frac{1}{8 \pi}(-1)^{\frac{l_{j}}{2}} \frac{1 \times 3 \times \ldots \times\left(l_{j}+1\right)}{2 \times 4 \times \ldots \times\left(l_{j}-2\right)} \hat{c}_{j} & j>1
\end{array}\right.
$$

where $l_{j}$ is the order associated to the $j$-th element of the modified $\mathrm{SH}$ basis (see [2, 3] for details). We emphasize that at each iteration, we estimate the same ODF as we would obtain by off-line processing.

\subsection{Motion detection}

The Kalman filter introduced in Section 2.1 reconstructs the spherical harmonics coefficients $\hat{\mathbf{c}}$ to best fit the signal $\ln (-\ln (E))$, for the $\ell_{2}$ norm. After a few iterations, the estimate $\hat{\mathbf{c}}_{k-1}$ is stable enough to predict with good accuracy the next signal outcome $y_{k}$.

However, if the subject moves within the scanner, the diffusion weighted images will no longer be registered to the baseline image, and pixels will not match from one volume to another. An abnormal increase in the prediction error of the diffusion signal during on-line reconstruction is a direct and expected consequence of patient motion. We propose to use this quantity as an indicator to warn the scanner operator to stop the scan in such a situation, as the next acquisitions would hardly be useful for ODF reconstruction. For each iteration, we calculate the prediction error $\epsilon_{k}=y_{k}-\mathbf{X}_{k} \hat{\mathbf{c}}_{k-1}$ at each voxel, and compute the average squared prediction error on the whole masked volume.

Next, we present results of simulated and in vivo fiber crossing detection, as well as motion detection. 
(a)

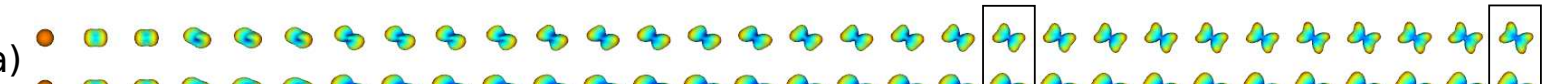

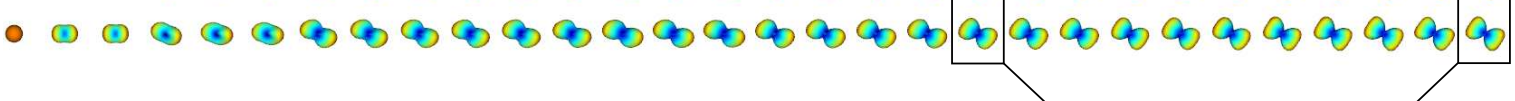

(b)

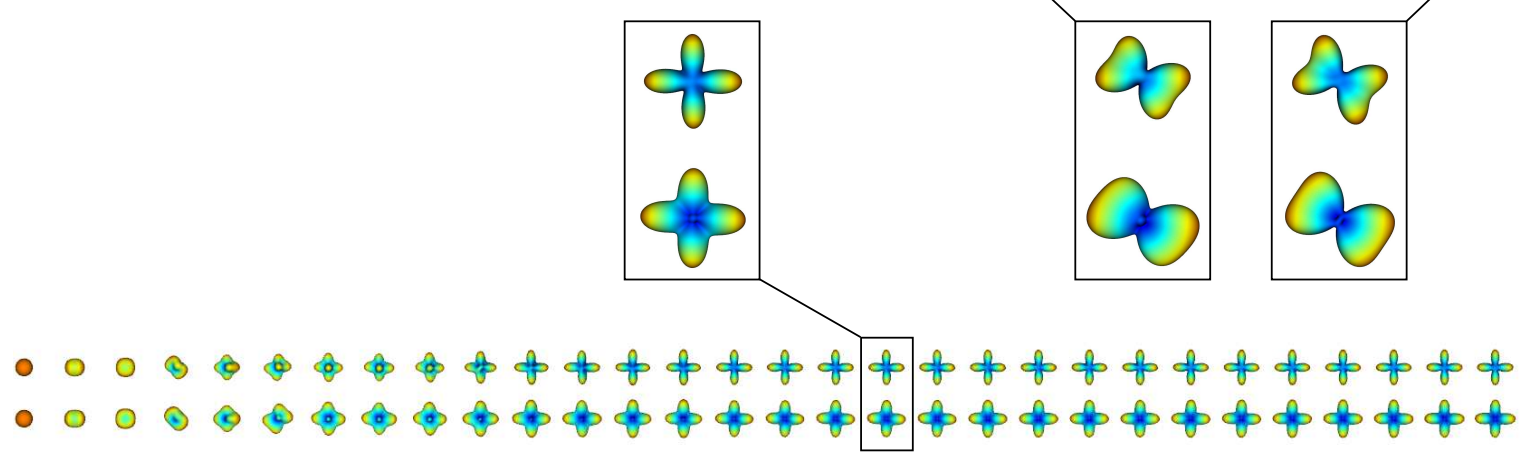

Fig. 1. Incrementally reconstructed ODFs from synthetic data with fiber crossings of (a) $60^{\circ}$ and (b) $90^{\circ}$, for iterations 1 to 30 (left to right), using (top) CSA QBI, and (bottom) original QBI with Laplace-Beltrami sharpening.

\section{EXPERIMENTS AND RESULTS}

\subsection{Results on artificial data}

We simulated fiber crossing by generating diffusion images from the sum of two exponentials:

$$
E(\hat{u})=\frac{1}{2}\left(e^{-\hat{u}^{T} \mathbf{D}_{1} \hat{u}}+e^{-\hat{u}^{T} \mathbf{D}_{2} \hat{u}}\right)
$$

where $\mathbf{D}_{1}$ is a diagonal matrix with diagonal entries $(9,2,2)$ and $\mathbf{D}_{2}$ is $\mathbf{D}_{1}$ rotated about the $y$-axis, once by $60^{\circ}$ and another time by $90^{\circ}$. Assuming an apparent diffusion coefficient (ADC) of $0.7 \times 10^{-3} \mathrm{~mm}^{2} / \mathrm{s}$ (the mean diffusivity in brain parenchyma), these diffusion values correspond to a $b$-value of $4800 \mathrm{~s} / \mathrm{mm}^{2}$. The ODFs were reconstructed in the fourth order SH basis using 30 iterations. The results are shown in Fig. 1 for CSA QBI, and also for original QBI followed by Laplace-Beltrami sharpening (I $-\alpha \nabla_{b}^{2}$ operator, see [12]), with parameter $\alpha=0.15$ chosen to produce the optimal results. CSA QBI was shown in [3] to resolve the fiber crossings starting at a smaller angle compared to the original QBI. Accordingly, we can see in Fig. 1 that the $60^{\circ}$ crossing is better resolved by the CSA ODF at most iterations, and the $90^{\circ}$ crossing is reconstructed equally well by both methods. Additionally, further experiments demonstrated that Kalman filtering in practice converges slightly faster when used with the CSA ODF than the original ODF (rate of convergence was smaller by 0.001 for crossing angles less than $50^{\circ}$, and about the same for larger angles).

\subsection{In vivo motion detection}

Images were acquired on 3T Siemens scanner at the Center for Magnetic Resonance Research, University of Minnesota,

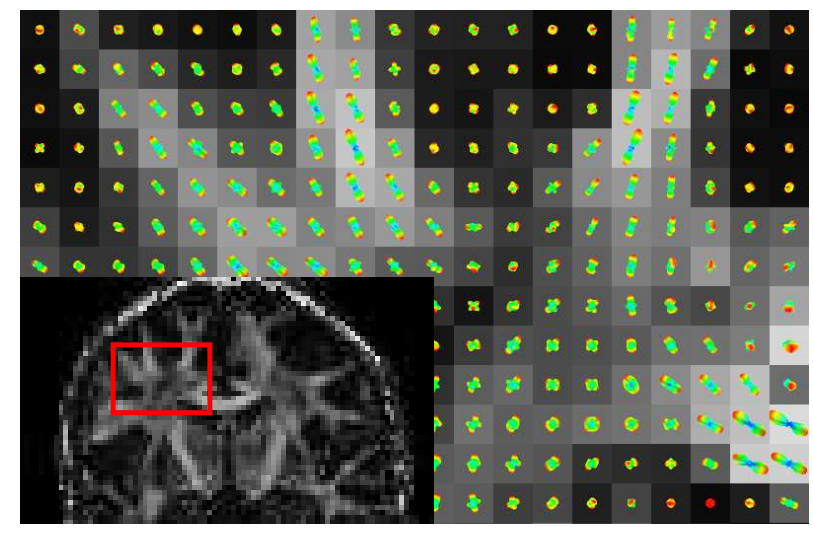

Fig. 2. Coronal slice: CSA ODF, after iteration 100 of the Kalman filter. The ODFs are shown on the tensor Fractional Anisotropy map.

with 200 encoding directions computed following the optimal sampling scheme of [7], $b=1000 \mathrm{~s} / \mathrm{mm}^{2}, 64$ slices with $2 \mathrm{~mm}$ thickness, $25 b=0 \mathrm{~s} / \mathrm{mm}^{2}$ images, $128 \times 128$ image matrix, TE $=90 \mathrm{~ms}$ and $\mathrm{TR}=8500 \mathrm{~ms}$. During the acquisition, the subject was asked to tilt his head after about 80 diffusion weighted images were acquired. A pilot sequence without motion was also acquired for comparison (see Fig. 2).

The motion was quantitatively investigated (but not corrected) through rigid registration of two baseline images acquired before and after the motion occured. We used the Slicer3 rigid registration module, which estimated the transformation to be a rotation of about $20^{\circ}$ around the $z$-axis, combined with a small translation. Axial views of both positions are shown in Fig. 3.

The Kalman filter of Section 2.1 was used to estimate the SH coefficients of the double logarithm of the signal, with a 

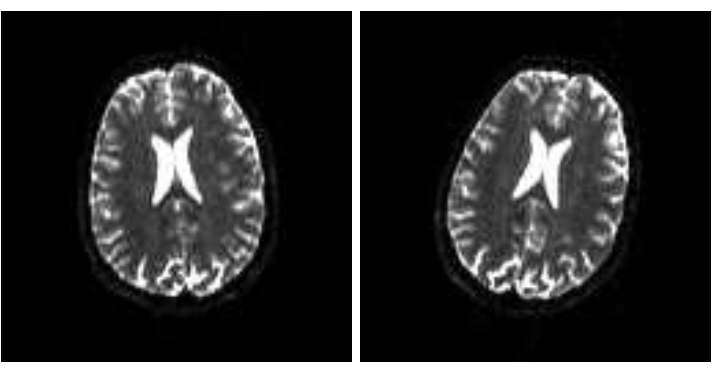

Fig. 3. Axial slices of two baseline images: (left) before and (right) after the motion occurred.

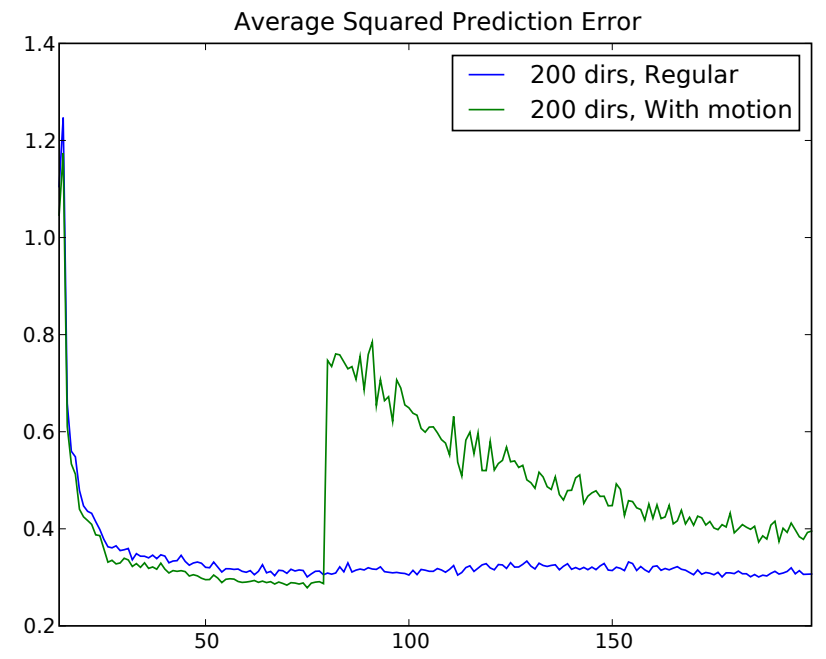

Fig. 4. Comparison of the average squared prediction error with and without motion.

regularization parameter $\lambda=0.006$. The voxel-wise squared prediction error was calculated at each iteration of the Kalman filter, and averaged over the relevant part of the imaging volume. This region of interest was defined by a simple thresholding of the baseline image.

In Fig. 4, we compare the evolution of the reconstruction error during both acquisition sequences. For the pilot acquisition, the fit gets better as new measurements are acquired, and the prediction error decreases, as expected. On the contrary, there is a sudden increase in prediction error for the second experiment, at a point corresponding exactly to the motion (iteration 80).

\section{CONCLUSION}

We have adapted and extended the on-line reconstruction algorithm to the mathematically correct CSA ODF; our Kalman filtering solution is shown to detect crossings in white matter along the acquisition and reconstruction process beyond what was possible with regular ODF. We have also studied local changes in diffusion signal induced by subject's motion, and have shown that motion could be detected on-line when a coherent increase in prediction error is reported over the imaging volume. We believe these improvements provide the clinicians with a more accurate and more flexible tool for QBI acquisition and analysis, in particular for children or patients who have difficulty sustaining prolonged scanning sessions.

The next challenging step towards these objectives could be the development of a motion compensation solution to combine with the Kalman filter. Real-time model estimation also opens the way for a wide variety of on-line postprocessing methods, such as scalar index computation or fiber tractography, giving valuable feedback to the operator.

Acknowledgements: This work was partly supported by the Computational Diffusion MRI (CD-MRI) INRIA Associate Team program, the OdysséeEADS Grant \#2118, NIH (P41 RR008079, P30 NS057091, R01 EB007813, R01 MH060662, R01 EB008432), ONR, NGA, NSF, DARPA and ARO.

\section{REFERENCES}

[1] D. S. Tuch, "Q-ball imaging," Magn. Reson. Med., vol. 52, no. 6, pp. 1358-1372, 2004.

[2] M. Descoteaux, E. Angelino, S. Fitzgibbons, and R. Deriche, "Regularized, fast, and robust analytical q-ball imaging," Magn. Res. Med., vol. 58 , no. 3 , pp. 497-510, 2007.

[3] I. Aganj, C. Lenglet, G. Sapiro, E. Yacoub, K. Ugurbil, and N. Harel, "Reconstruction of the orientation distribution function in single and multiple shell q-ball imaging within constant solid angle," Magn. Reson. Med., 2009, in press.

[4] A. Tristán-Vega, C.-F. Westin, and S. Aja-Fernández, "Estimation of fiber orientation probability density functions in high angular resolution diffusion imaging," NeuroImage, vol. 47, no. 2, pp. 638-650, 2009.

[5] G. K. Rohde, A. S. Barnett, P. J. Basser, S. Marenco, and C. Pierpaoli, "Comprehensive approach for correction of motion and distortion in diffusion-weighted MRI," Magn. Reson. Med., vol. 51, no. 1, pp. 103114, January 2004.

[6] C. Poupon, A. Roche, J. Dubois, J.-F. Mangin, and F. Poupon, "Realtime MR diffusion tensor and q-ball imaging using Kalman filtering," Med. Image Anal., vol. 12, no. 5, pp. 527-534, 2008, Special issue on the 10th International Conference on MICCAI 2007.

[7] R. Deriche, J. Calder, and M. Descoteaux, "Optimal real-time q-ball imaging using regularized Kalman filtering with incremental orientation sets," Med. Image Anal., vol. 13, no. 4, pp. 564-579, August 2009.

[8] P. Funk, "Über eine geometrische Anwendung der Abelschen integralgleichung," Mathematische Annalen, vol. 1, no. 77, pp. 129-135, 1915.

[9] A. W. Anderson, "Measurement of fiber orientation distributions using high angular resolution diffusion imaging.," Magn. Reson. Med., vol. 54, no. 5, pp. 1194-1206, November 2005.

[10] C. P. Hess, P. Mukherjee, E. T. Han, D. Xu, and D. B. Vigneron, "Qball reconstruction of multimodal fiber orientations using the spherical harmonic basis.," Magn. Reson. Med., vol. 56, no. 1, pp. 104-117, July 2006.

[11] R. E. Kalman, "A new approach to linear filtering and prediction problems," Journal of Basic Engineering, vol. 82, no. 1, pp. 35-45, 1960.

[12] M. Descoteaux, E. Angelino, S. Fitzgibbons, and R. Deriche, "A linear and regularized ODF estimation algorithm to recover multiple fibers in q-ball imaging," Research Report 5768, INRIA, 2005. 